\title{
Efficacy of anti-inflammatory drug ulinastatin in coronavirus disease 2019: A case report
}

\author{
Rupa Chatterjee1, ShahafazIbi Meethal Puthiya Purayil ${ }^{1}$, Raneesh Kumar Ramakrishnan ${ }^{2}$, \\ Rajesh Sankaran', Changam Sheela Sasikumar ${ }^{3}$ \\ From ${ }^{1}$ Junior Medical Resident, ${ }^{2}$ Director and Intensivist, Department of Covid Ward, Hycare Superspeciality Hospital, ${ }^{3}$ Head, Department of Clinical \\ Research, Hycare Superspeciality Hospital, Chennai, Tamil Nadu, India
}

\begin{abstract}
Coronavirus disease 2019 (COVID-19), caused by severe acute respiratory coronavirus 2, can affect the respiratory system as well as other systems of the body. It has now become a pandemic and poses a global health emergency. We report the case of a COVID-19positive 50-year-old male patient with multiple, ground-glass opacities in bilateral lungs. The disease did not respond well to tocilizumab administration and the condition of the patient gradually worsened. We administered ulinastatin along with high doses of steroids. We observed that the overall performance of a combination of ulinastatin and high-dose steroids proved to be effective and routine use of ulinastatin is a potent method of treatment in COVID-19 patients developing acute respiratory distress syndrome, which may save other patients with a similar background.
\end{abstract}

Key words: Anti-inflammatory, Coronavirus disease 2019, Ulinastatin

$\mathrm{C}$ oronavirus disease 2019 (COVID-19), caused by severe acute respiratory coronavirus 2 (SARS-CoV-2), can affect the respiratory system as well as other systems of the body [1]. It has now become a pandemic and poses a global health emergency [2]. While the majority of patients with COVID-19 have mild-to-moderate symptoms, approximately $14 \%$ of the patients may progress to severe pneumonia and exhibit considerable fatality [3]. To date, there are no specific therapeutic agents or vaccines available. Immunologic characteristics of patients with severe COVID-19 exhibit remarkably elevated serum levels of pro-inflammatory cytokines including interleukin-6 (IL-6), tumor necrosis factor- $\alpha$ (TNF- $\alpha)$, and IL-1 $\beta$, characterized as a cytokine storm. The cytokine storm is believed to play a critical role in COVID-19 progression, deterioration, and even death [3]. Strategies to dampen the inflammatory responses are, therefore, proposed.

Ulinastatin is a glycoprotein extracted from the fresh human urine. It inhibits the activity of various proteolytic enzymes and has been widely used for the treatment of acute pancreatitis (AP). Meanwhile, ulinastatin has been demonstrated as an important anti-inflammatory and antioxidation agent and has been clinically used as a potential treatment for circulatory shock, severe sepsis,

\section{Access this article online}

Received - 19 September 2020

Initial Review - 04 October 2020

Accepted - 21 October 2020

DOI: $10.32677 /$ IJCR.2020.v06.i10.018 and acute respiratory distress syndrome (ARDS) [4-6]. Assuming the same pathophysiology to be functional in the ARDS of COVID-19 pneumonia, ulinastatin could limit the inflammation of the alveolar membrane and the systemic inflammatory response, thereby having a major role in the recovery of patients with severe and critical respiratory symptoms. We report the case of a COVID-19-positive 50-year-old male patient who was successfully treated with a combination of ulinastatin and highdose steroids.

\section{CASE REPORT}

A 50-year-old obese male with no known comorbidities was admitted to a hospital of South India with complaints of fever, cough, and shortness of breath for 5 days. The patient was tested COVID positive with computed tomography (CT). COVID reverse transcription polymerase chain reaction (RT-PCR) done outside revealed a negative result but CT showed CO-RADS-5 with multiple ground-glass opacities in bilateral lungs involving all lobes. The medical history and family history of the patient were non-significant.

On examination, he recorded a temperature of 101 degrees Fahrenheit, blood pressure of $110 / 80 \mathrm{mmHg}$, pulse rate of 100/min, respiratory rate of 52 (tachypnea), and oxygen saturation in room air

Correspondence to: Dr. Rupa Chatterjee, Hycare Superspeciality Hospital, No 37\&38 Razack Garden Road MMDA Colony, Arumbakkam, Chennai - 600 106, Tamil Nadu, India. E-mail: chatterjee.rupa@gmail.com

(C) 2020 Creative Commons Attribution-NonCommercial 4.0 International License (CC BY-NC-ND 4.0). 
was $72 \%$ which increased up to $86 \%$ with 151 of oxygen through non-rebreather face mask (NRBM). Respiratory system examination revealed decreased air entry in the bilateral lungs with crept.

According to the Indian guidelines [7] and based on the severity of the disease, the patient was initiated on inj. methylprednisolone $80 \mathrm{mg}$ Q8h, inj. remdesivir $100 \mathrm{mg}$ OD after a loading dose of $200 \mathrm{mg}$ on day 1, inj. low-molecular-weight heparin $0.6 \mathrm{mg}$ Q12h along with inj. tocilizumab $200 \mathrm{mg}$ Q12h (2 doses), inj. piperacillin + tazobactam $4.5 \mathrm{~g}$ Q6h, inj. doxycycline $100 \mathrm{mg}$ Q12h, T. azithromycin $500 \mathrm{mg}$ OD, T. Vitamin C $500 \mathrm{mg}$ OD, and T. zinc $20 \mathrm{mg}$ Q12h.

Investigations show the following values: IL- $6=39.36$, ferritin $=557.00 \mu \mathrm{g} / \mathrm{L}$, and $\mathrm{D}$-dimer $=0.50 \quad \mathrm{mcg} / \mathrm{mL}$. On the following day, in view of his persistent tachypnea, he was commenced on intermittent bilevel positive airway pressure $(\mathrm{I} / \mathrm{E}=18 / 12)$ with intermittent $15 \mathrm{~L}$ of oxygen through NRBM. On serial chest X-ray (CXR) monitoring, there was a worsening of bilateral heterogeneous opacities on the right side but the clinical condition of the patient remained the same with no fever spikes, respiratory rate maintaining at $38-40$, and saturation maintaining at $88 \%$ with oxygen support (Fig. 1a). After 2 days, COVID RT-PCR was repeated which revealed a positive result. On the $5^{\text {th }}$ day of admission to the hospital, a CXR was done (Fig. 1b) and investigations revealed values of IL- $6=526.8$, ferritin $=544.10 \mu \mathrm{g} / \mathrm{L}$, and D-dimer $=2.26 \mathrm{mcg} / \mathrm{mL}$.

Medications were revised and inj. ulinastatin, 200,000 units in $100 \mathrm{ml} \mathrm{NS}$ i.v Q8h, was added and inj. remdesivir was withheld. The condition of the patient improved in terms of respiratory rate (26-28/min) with a reduction in the oxygen dependency (4-6 L through NRBM) to maintain a saturation above $95 \%$. On the $9^{\text {th }}$ day, serial CXR monitoring was done which revealed resolution of the pneumonitic patches to an extent with reemergence of air pockets (Fig. 2a).

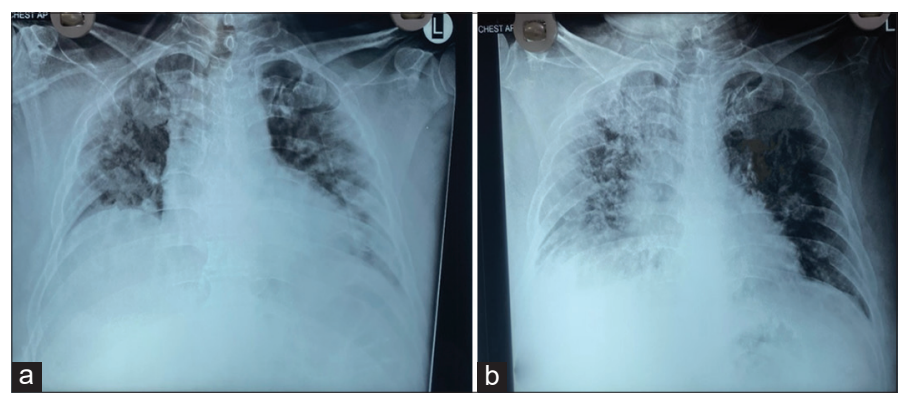

Figure 1: Chest X-ray done on (a) day 1 and (b) day 6
After 10 days, the CXR was performed again (Fig. 2b) and the investigations such as IL6 $=85.92$, ferritin $=197.50 \mathrm{mg} / 1$, and D-dimer $0.12 \mathrm{mcg} / \mathrm{mL}$ were done. Henceforth, the frequency of administration inj. ulinastatin was gradually reduced from 200,000 units Q8h and was withheld after a total of 9 days of administration. After 2 weeks, the CXR was done (Fig. 2c) and the patient was counseled for deep breathing and other respiratory exercises. After 20 days of admission, the patient was discharged maintaining a respiratory rate of $22 / \mathrm{min}$ and saturation of $90 \%$ at room air. Post-discharge instructions were given to the patient.

\section{DISCUSSION}

The cytokine storm is believed to play an essential role in the pathogenesis of COVID-19 and might be correlated with disease severity and fatality. It is thought to be the reason for rapid multiorgan failure [8]. The study of SARS-CoV showed that virus-infected lung epithelial cells produced IL-8 in addition to IL-6. IL-8 is a well-known chemoattractant for neutrophils and $\mathrm{T}$ cells. Infiltration of a large number of inflammatory cells was observed in the lungs from severe COVID-19 patients and these cells presumably consist of a constellation of innate immune cells and adaptive immune cells. Therefore, modulation of the immune response or suppression of over-reactive cytokine production may prove crucial in severe cases [9].

Ulinastatin, an intrinsic broad-spectrum protease inhibitor, could effectively inhibit a variety of cell proteolytic enzymes and have multifunctional therapeutic mechanisms. First, ulinastatin has an inhibitory effect on the production of inflammatory cytokines and adhesion molecules [10]. Meanwhile, ulinastatin improves the stability of the lysosomal membrane and reduces the synthesis and delivery of lysosomal enzymes, thus scavenging oxygen or hydroxyl radicals. Treatment with ulinastatin reduces the level of TNF- $\alpha$ and IL-1 $\beta$ in a dose-dependent way. TGF$\beta 1$ and IL-10 are T-cell-related cytokines characterized as immunosuppressive or anti-inflammatory mediators. The previous studies have shown that ulinastatin can inhibit the expression of TNF- $\alpha$ and IL- $1 \beta$, and increase the levels of IL-2 and IL-10 [11,12]. The studies on the mechanism of the antiinflammatory effect of ulinastatin have mainly been focused on the roles of these cytokines. A recent meta-analysis of 33 randomized controlled trials involving 2344 patients of ARDS showed that as compared to conventional therapy, ulinastatin was

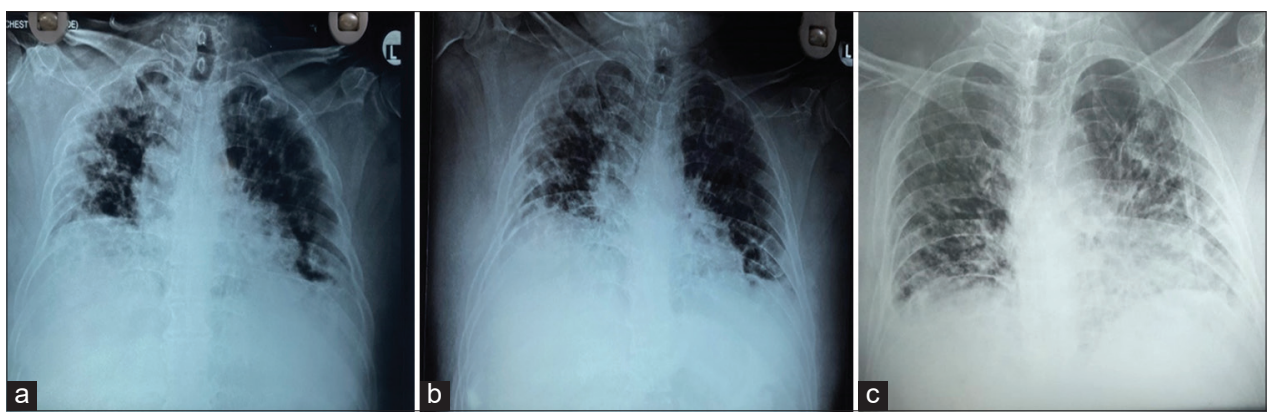

Figure 2: Chest X-ray done on (a) day 9; (b) day 11; (c) day 14 
superior in reducing mortality, ventilator-associated pneumonia, duration of mechanical ventilation, length of hospital stay, and increasing the patients oxygenation index. The meta-analysis had also demonstrated a significant reduction in levels of TNF$\alpha$, IL-1 $\beta$, IL-6, and IL-8 [6]. A consensus document from China has recommended high-dose ulinastatin in the prevention and management of cytokine storm in patients with COVID-19 [13].

In recent years, evidence has accumulated that immune reactions and immune cells such as CD4 $+\mathrm{T}$ cells and Tregs play important roles in the AP pathogenesis [14,15]. Hence, inhibiting pro-inflammatory mediators $[16,17]$ and regulating immune reactions are important considerations in the therapy of severe AP [15]. It has been confirmed that ulinastatin can regulate the immunological function through these special immune cells $[18,19]$. The pro-inflammatory cytokines such as IL-1 $\beta$, IL-10, and TNF- $\alpha$ have been shown to play an important role in the pathogenesis of AP causing tissue damage and organ dysfunction. In the course of AP, multiorgan damage caused in the early stage associated with systemic inflammatory response, followed by pancreatic necrosis in the late stage are the two most important events responsible for mortality [20]. The intervention in the early stage by targeting an inflammatory response may be an effective treatment strategy in the treatment of AP [21].

\section{CONCLUSION}

A high dose of ulinastatin treatment is safe and potentially beneficial for the patients with COVID-19, with rapid improvement of clinical symptoms, blood parameters, and absorption of the pulmonary lesions.

\section{REFERENCES}

1. Guan WJ, Ni ZY, Hu Y, Liang WH, Ou CQ, He JX, et al. Clinical characteristics of coronavirus disease 2019 in China. N Engl J Med 2020;382:1708-20.

2. Cucinotta D, Vanelli M. WHO declares COVID-19 a pandemic. Acta Biomed 2020;91:157-60.

3. Yang XB, Yu Y, Xu JQ, Shu HQ, Xia JA, Liu H, et al. Clinical course and outcomes of critically ill patients with SARS-CoV-2 pneumonia in Wuhan, China: A single-centered, retrospective, observational study. Lancet Respir Med 2020;8:475-81.

4. Karnad DR, Bhadade R, Verma PK, Moulick ND, Daga MK, Chafekar ND, et al. Intravenous administration of ulinastatin (human urinary trypsin inhibitor) in severe sepsis: A multicenter randomized controlled study. Intensive Care Med 2014;40:830-8.

5. Ji M, Chen T, Wang B, Chen M, Ding Q, Chen L, et al. Effects of ulinastatin combined with mechanical ventilation on oxygen metabolism, inflammation and stress response and antioxidant capacity of ARDS. Exp Ther Med 2018:15:4665-70.

6. Zhang X, Zhu Z, Jiao W, Liu W, Liu F, Zhu X. Ulinastatin treatment for acute respiratory distress syndrome in China: A meta-analysis of randomized controlled trials. BMC Pulm Med 2019;19:196.

7. Ministry of Health and Family Welfare, Directorate General of Health Services, Government of India. Revised Guidelines on Clinical Management of COVID-19. Available from: https://www.mohfw.gov.in/ pdf/RevisedNationalClinicalManagementGuidelineforCOVID1931032020. pdf. [Last accessed on 2020 Sep 10].

8. Mehta P, McAuley DF, Brown M, Sanchez E, Tattersall RS, Manson JJ. COVID-19: Consider cytokine storm syndromes and immunosuppression. Lancet 2020;395:1033-4.

9. Zhou D, Dai SM, Tong Q. COVID-19: A recommendation to examine the effect of hydroxychloroquine in preventing infection and progression. $\mathrm{J}$ Antimicrob Chemother 2020;75:1667-70.

10. Kanai T, Ishiwata T, Kobayashi T, Sato H, Takizawa M, Kawamura Y, et al. Ulinastatin, a urinary trypsin inhibitor, for the initial treatment of patients with Kawasaki disease: A retrospective study. Circulation 2011;124:2822-8.

11. Liu R, Qi H, Wang J, Wang Y, Cui L, Wen Y, et al. Ulinastatin activates the renin-angiotensin system to ameliorate the pathophysiology of severe acute pancreatitis. J Gastroenterol Hepatol 2014;29:1328-37.

12. Ma T, Kang C, Shao H, Qi Q, Hu W. Protective effects of ulinastatin on proliferation and cytokine release of splenocytes from rats with severe acute pancreatitis. Eur Surg Res 2006;38:445-50.

13. Shanghai Novel Coronavirus Disease Clinical Treatment Expert Group. Expert consensus on comprehensive treatment of 2019 novel coronavirus disease in Shanghai Chin J Infect Dis 2020;1:38.

14. Zheng L, Xue J, Jaffee EM, Habtezion A. Role of immune cells and immunebased therapies in pancreatitis and pancreatic ductal adenocarcinoma. Gastroenterology 2013;144:1230-40.

15. Shamoon M, Deng Y, Yong QC, Bhatia M, Jia S. Therapeutic implications of innate immune system in acute pancreatitis. Expert Opin Ther Targets 2016;20:73-87.

16. Bhatia M, Brady M, Zagorski J, Christmas SE, Campbell F, Neoptolemos JP, et al. Treatment with neutralising antibody against cytokine induced neutrophil chemoattractant (CINC) protects rats against acute pancreatitis associated lung injury. Gut 2000;47:838-44.

17. Bhatia M, Proudfoot AE, Wells TN, Christmas S, Neoptolemos JP, Slavin J. Treatment with Met-RANTES reduces lung injury in caerulein-induced pancreatitis. Br J Surg 2003;90:698-704.

18. Hao X, Han J, Xing Z, Hao Y, Jiang C, Zhang J, et al. Urinary trypsin inhibitor attenuated inflammatory response of patients undergoing cardiopulmonary bypass by inducing activated Treg cells. Inflammation 2013;36:1279-85.

19. Zhang L, Wang N, Zhou S, Ye W, Yao Q, Jing G, et al. Preventive effect of ulinastatin on postoperative complications, immunosuppression, and recurrence in esophagectomy patients. World J Surg Oncol 2013;11:84.

20. Minkov GA, Halacheva KS, Yovtchev YP, Gulubova MV. Pathophysiological mechanisms of acute pancreatitis define inflammatory markers of clinical prognosis. Pancreas 2015;44:713-7.

21. Sendler M, Dummer A, Weiss UF, Kr BG, Wartmann T, Scharffetter KK, et al. Tumour necrosis factor $\alpha$ secretion induces protease activation and acinar cell necrosis in acute experimental pancreatitis in mice. Gut 2013;62:430-9.

Funding: None; Conflicts of Interest: None Stated.

How to cite this article: Chatterjee R, Purayil SMP, Ramakrishnan RK, Sankaran R, Sasikumar CS. Efficacy of anti-inflammatory drug ulinastatin in coronavirus disease 2019: A case report. Indian J Case Reports. 2020;6(10):601-603. 